\title{
Mobility and Reactivity of Oxygen Adspecies on Platinum Surface
}

Wei Wang, Jie Zhang, Fangfang Wang, Bing-Wei Mao, Dongping Zhan*, Zhong-Qun Tian

State Key Laboratory of Physical Chemistry of Solid Surfaces, Collaborative Innovation Center of Chemistry for Energy Materials, and Department of Chemistry, College of Chemistry and Chemical Engineering, Xiamen University, Xiamen 361005, China 


\section{Contents}

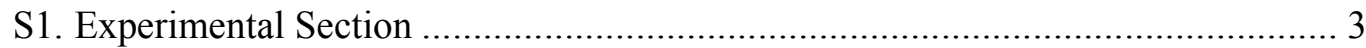

S2. Fabrication and preparation of Pt Nanoelectrodes ............................................ 4

S3. Preparations of the 5- $\mu$ m-radius Pt ultramicroelectrodes ................................... 5

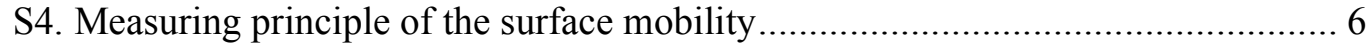

S5. Further discussion on surface diffusion.......................................................... 10

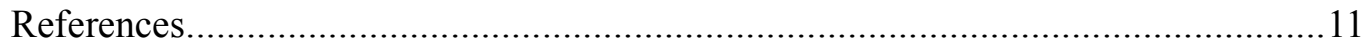




\section{S1. Experimental Section}

Chemicals Hexaammineruthenium (III) chloride $\left(\mathrm{Ru}\left(\mathrm{NH}_{3}\right)_{6} \mathrm{Cl}_{3}, 99 \%\right)$ and ferrocenemethanol are purchased from Sigma-Aldrich. $\mathrm{H}_{2} \mathrm{SO}_{4}$ and $\mathrm{KCl}$ are provided by Sinopharm Group Co. Ltd. and used without further purification. Aqueous solutions were prepared from deionized water (18.2 M $\Omega \mathrm{cm}$, Milli-Q, Millipore Co.).

Measurements and Instruments The apparent size of $\mathrm{Pt}$ nanoelectrode was evaluated by steady-state voltammetry in an aqueous solution with $2 \mathrm{mM}$ $\mathrm{Ru}\left(\mathrm{NH}_{3}\right)_{6} \mathrm{Cl}_{3}$ and $100 \mathrm{mM} \mathrm{NaCl}$, while an $\mathrm{Ag} / \mathrm{AgCl}$ wire was used as both the reference and counter electrode. Only the electrodes producing defined sigmoidal voltammograms were used for further experiments. Experiments in $\mathrm{H}_{2} \mathrm{SO}_{4}$ solution were performed with a saturated $\mathrm{Hg} / \mathrm{Hg}_{2} \mathrm{SO}_{4}$ reference electrode (MSE) to avoid the interference of $\mathrm{Cl}^{-}\left(\mathrm{E}_{\mathrm{MSE}}=0.64 \mathrm{~V}\right.$ vs. RHE). Cyclic voltammograms were obtained by using PGSTAT302N Autolab (Metrohm Autolab B.V., The Netherlands). During the experiments, the solutions were purged with high-purity argon before and during the experiments to remove oxygen. All these experiments were performed at room temperature $(298 \mathrm{~K})$ in a Faraday cage. For the measurement of pre-exponential factor $\left(D_{0}\right)$ and activation energies $\left(\Delta E_{d}\right)$, experiments were carried out between 303 and 333 $\mathrm{K}$ with an interval of $10 \mathrm{~K}$ using a continuous flow cryostat at a constant temperature and in a deoxidized cell. Before the CO stripping voltammetry, a 5- $\mu \mathrm{m}$-radius Pt UME was adopted to enhance the current response. The Pt UME was activated in the potential region from $-0.04 \mathrm{~V}$ to $1.54 \mathrm{~V}$ (vs. MSE) with a scanning rate of $0.5 \mathrm{~V} \cdot \mathrm{s}^{-1}$ for 200 cycles. Then, $\mathrm{CO}$ is pre-adsorbed on the Pt UME at a constant potential of $0.1 \mathrm{~V}$ while the solution is bubbled with CO gas. Scanning electron microscopy (SEM) images of the nanoelectrodes are obtained on a HITACHI S-4800 scanning electron microscope (Hitachi High-Technologies, Co.). 


\section{S2. Fabrication and preparation of Pt Nanoelectrodes}

As described previously ${ }^{1-3}$, the fabrication of Pt nanoelectrodes (Goodfellow Co., UK) consists of six steps which are exhibited in Fig. S1. Firstly, a 25- $\mu$ m-diameter Pt wire is placed at the middle of a borosilicate capillary (Drummond Co. USA; $1.0 \mathrm{~mm}$ o.d., $0.2 \mathrm{~mm}$ i.d.). With a programmed laser-beam heating puller (P-2000, Sutter Co., USA), the middle part of the capillary was pre-thinned into an hourglass shape. The metal wire was sealed into the capillary under vacuum with the second heating process. During the third heating process, the micropipette was pulled violently and broken as two glassing-dealing nanoelectrodes. A 100- $\mu$ m-diameter Ni wire was used as the electrical connector of the nanoelectrode. Due to the difference of the ductility between glass and metal wire, the wire was normally sealed in glass at the tip of the nanoelectrode. We used the Sutter BV-10 beveller (Sutter Co., USA) with a $50 \mathrm{~nm}$ lapping tape to expose the metal disc. The whole process was monitored under a video microscopic.

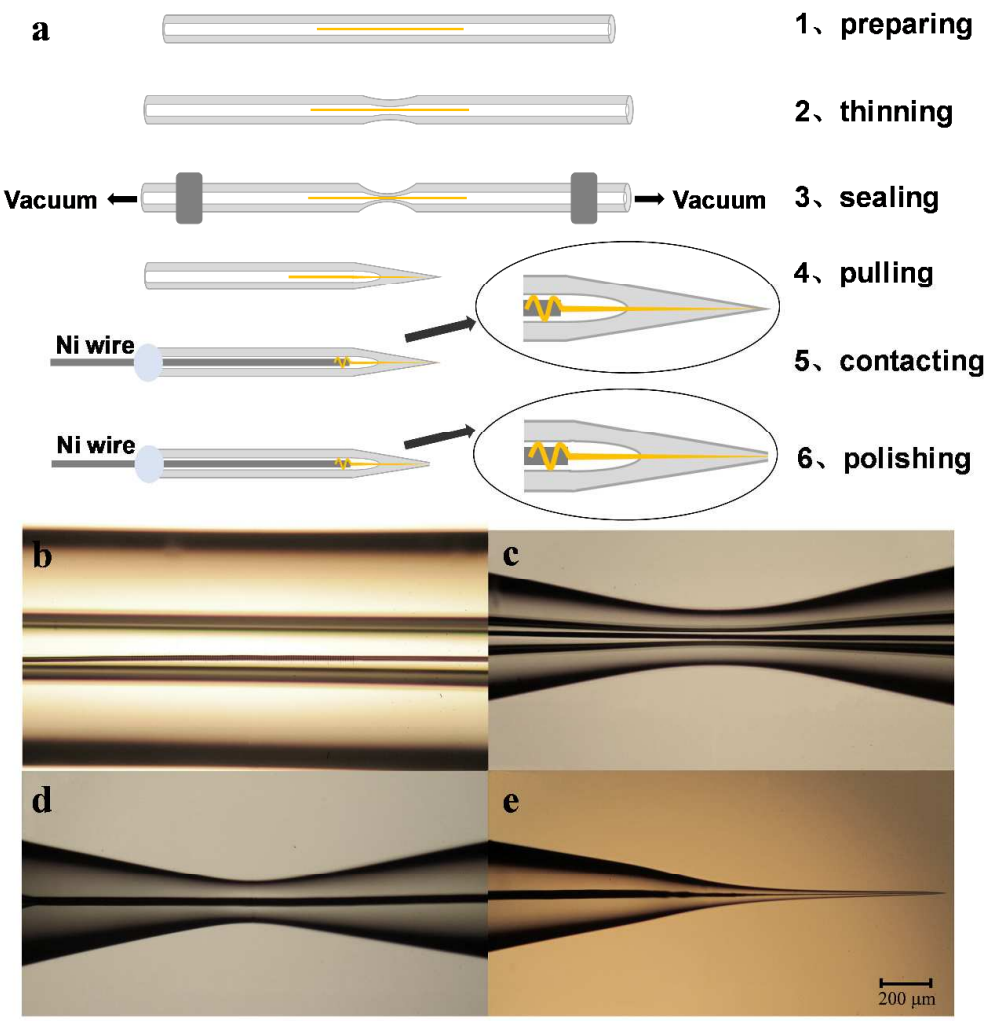

Figure S1 Fabrication of Pt nanoelectrodes. (a) The schematic protocols for the fabrication of nanoelectrodes. (b)-(d), The optical micrographs of the nanoelectrode during fabrication step 1-4: (b) preparing, (c) thinning, (d) sealing, (e) pulling. 


\section{S3. Preparations of the 5- $\mu$ m-radius Pt ultramicroelectrodes}

Figure S2 presents the details of the preparation of Pt ultramicroelectrodes (UME). An annealed 5- $\mu$ m-radius Pt wire (Goodfellow, 99.99\%) was attached to a $100 \mu \mathrm{m}$ nickel (Ni) wire using Ag conductive epoxy. The Pt wire with Ni connector was then inserted into a borosilicate capillary ( $2.0 \mathrm{~mm}$ o.d., $0.7 \mathrm{~mm}$ i.d.), leaving $0.5 \mathrm{~cm} \mathrm{Pt}$ wire outside the capillary. Epoxy resin (LECO) was then inhaled into the end of the capillary to seal the Pt wire. A copper wire Electrical was connected with the nickel wire by Ag conductive epoxy. The Pt UME was polished with emery paper of decreasing grain size followed by alumina powder with 1 and $0.3 \mu \mathrm{m}$ particle sizes in sequence.
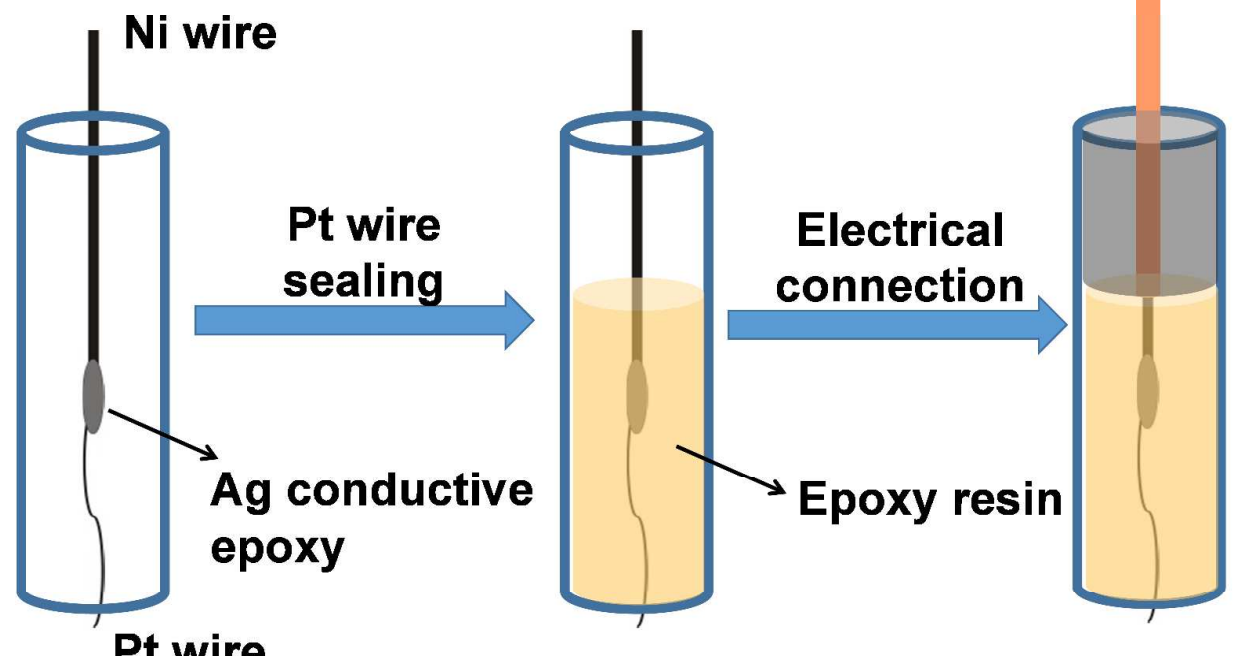

Figure S2 The schematic protocols for the preparation of Pt UME sealed in epoxy. 


\section{S4. Measuring principle of the surface mobility}

Figure 2 in the text depicts that the glass-sealing Pt nanoelectrodes can separate the Faraday adsorption and desorption processes from the surface mobility spatially. In fact, the Faradaic adsorption and desorption processes occur at the platinum (Pt) nanoelectrode/electrolyte interface, and the surface diffusion occurs along the side face of the adjacent $\mathrm{Pt}$ wire. Because the size of the Pt/electrolyte interface are reduced to nanometer scale, the relative contribution to the integral charge of Faradaic desorption at the Pt nanoelectrode/electrolyte interface becomes smaller, thus, the contribution of surface diffusion is prominent. It should be noted that the total current response comes from three parts: the pseudocapacitor of the electric double layer, the Faradaic desorption of oxygen adsorbates at the Pt nanoelectrode/electrolyte interface, and the Faradaic desorption of the diffused oxygen adspecies at the adjacent Pt wire surface.

For the charge and discharge of the electric double layer, the current can be expressed as:

$$
i_{c}=C_{d} v
$$

where, $i_{c}$ is the charging and discharging current of electric double layer, $C_{d}$ is the pseudocapacitor of electric double layer, $v$ is the scan rate of cyclic voltammetry. Thus, the integral charge caused by the charge and discharge of electric double layer, $Q_{c}$, can be expressed as:

$$
Q_{c}=\int i_{c} d t=\int C_{d} v d t=\int C_{d} \frac{d E}{d t} d t=\int C_{d} d E=C_{d} \Delta E
$$

Suppose the Faraday desorption of oxygen adsorbates on Pt are kinetically fast, the current can be expressed:

$$
i=-n F A \frac{d \Gamma^{*}}{d t}
$$

Where, $i_{p}$ is the peak current of cyclic voltammogram, $n$ the transferred charge, $F$ the Faraday constant, $A$ the electrode area, $\Gamma^{*}$ the surface concentration of the adsorbates, $R$ the gas constant, $T$ the absolute temperature, $v$ the scanning rate. Thus, the integral charge caused by the Faraday desportion in the region of the nanoelectrode/electrolyte interface, $Q_{a}$, can be expressed as ${ }^{4}$ : 


$$
Q_{a}=\int-n F A \frac{d \Gamma^{*}}{d t} d t=-n F A \Gamma^{*}
$$

As depicted in Fig. 2 in the main text, the surface diffusion of oxygen adsorbates is actually in a simple one-dimensional diffusion field which can be described by Fick' second law:

$$
\frac{\partial C}{\partial t}=\frac{\partial^{2} C}{\partial x^{2}}
$$

The boundaries are listed as followed:

$$
\begin{array}{ll}
t=0, x=0, & C=F C^{*} \\
t>0, x=0, & C=f(E) \\
t \geq 0, x \rightarrow \infty, & C=0
\end{array}
$$

As mentioned above, the Faraday desorption of oxygen adsorbates on Pt surface are kinetically fast, it is reasonable to consider that the surface diffusion of oxygen adsorbates is the rate determining rate. The Faraday current caused by surface diffusion can be expressed as: ${ }^{4}$

$$
i=n F p(n F / R T)^{1 / 2} C^{*} D^{1 / 2} v^{1 / 2} \pi^{1 / 2} \chi(\sigma t)
$$

Where, $p$ is perimeter of the disc nanoelectrode, $C^{*}$ is the saturate surface concentration of oxygen adsorbates, details of $\chi(\sigma t)$ can be check in literature ${ }^{5}$. The integral charge caused by surface diffusion, $Q_{d}$, can be expressed as:

$$
Q_{d}=\left[n F p\left(\frac{n F}{R T}\right)^{1 / 2} C^{*} D^{1 / 2} \pi^{1 / 2} \int_{E_{1}}^{E_{2}} \chi(\sigma t) d E\right] v^{-1 / 2}
$$

From the above analysis, it can be conclude that $Q_{c}$ and $Q_{a}$ are independent of scan rate, however, $Q_{d}$ is in reverse proportion to the square root of scan rate (Fig. S3). The surface diffusion coefficient can be derived from the slope while the roughness factor (i.e., the ratio of "real area" over geometric area) can be derived from the intercept at the infinite scan rate. This principle is depicted in Fig. S3 as well as 
Figure 2 in the text. Besides the data shown in the main text, a group of experimental data for Pt nanoelectrodes is shown in Fig. S4.

For measuring the activation energy $\Delta E_{d}$ and the prefactor $D_{0}$ of surface diffusion based on Arrhenius equation, investigations were carried out at different temperature. These experiments are performed in a special electrochemical cell with a heat preservation chamber (Figure S5). As show in Fig. S6, the integral desorption charge is found in a reverse proportion to the square root of scan rate at each temperatures.
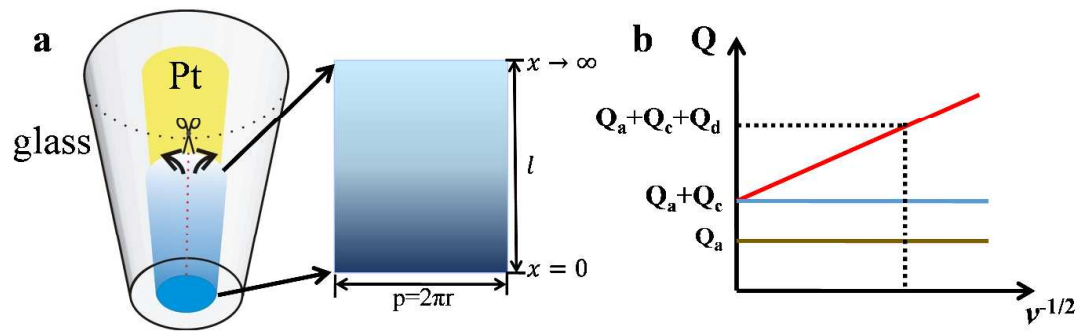

Figure S3 Schematic diagram of the model for measuring surface diffusion coefficient of oxygen adsorbates on Pt nanoelectrode. (a) Profile chart of the surface diffusion area; (b) The linear relationship between $Q$ and $v^{-1 / 2}$. The intercept is the integral charge caused by electric double-layer capacitor and oxygen desorption at the nanoelectrode/electrolyte interface, which reflects the electrocatalytic activity, i.e., "real area", of the nanoelectrode. From the slope, the surface diffusion coefficient of oxygen adsorbates can be obtained.
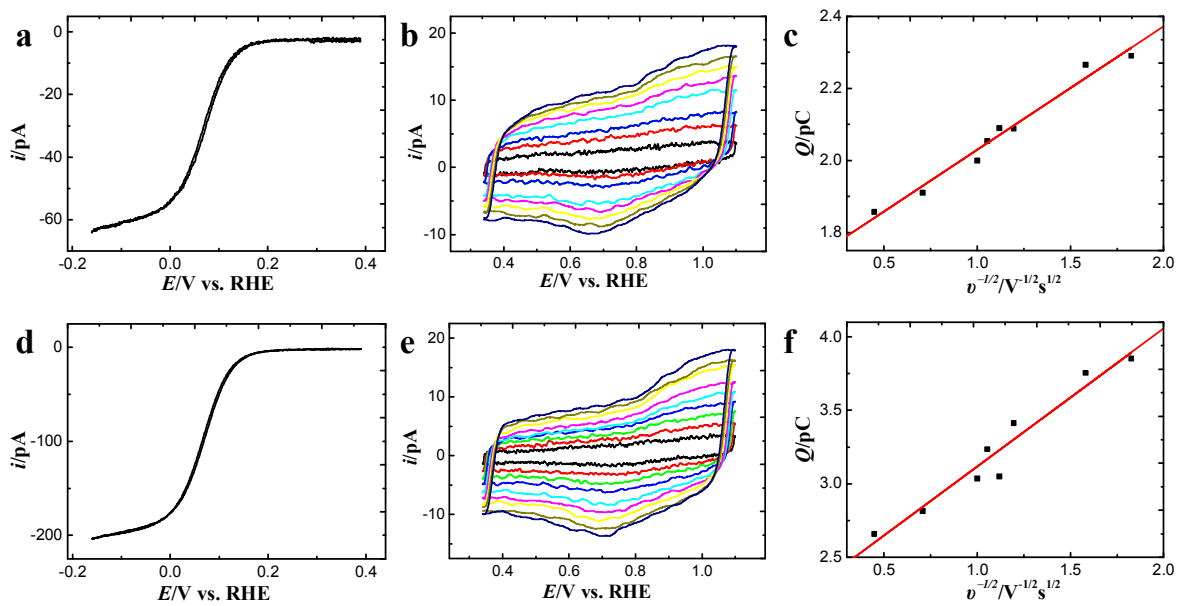

Figure S4 Measurements of surface diffusion coefficient of oxygen adspecies on $\mathrm{Pt}$ nanoelectrodes. (a, d) Steady state voltammograms obtained at Pt nanoelectrodes with different radius in a solution with $2 \mathrm{mM} \mathrm{Ru}\left(\mathrm{NH}_{3}\right)_{6} \mathrm{Cl}_{3}$ and $0.1 \mathrm{M} \mathrm{KCl}$, (a) $141 \mathrm{~nm}$, (d) $470 \mathrm{~nm}$. scan rates are $0.05 \mathrm{~V} \cdot \mathrm{s}^{-1}$. (b) and (e) are the cyclic voltammograms obtained at the corresponding Pt nanoelectrodes in $0.5 \mathrm{M} \mathrm{H}_{2} \mathrm{SO}_{4}$ solution deoxygenated with Ar gas, the scan rates were $0.1,0.2,0.3,0.4,0.5,0.6,0.7,0.8,0.9$ and $1 \mathrm{~V} \cdot \mathrm{s}^{-1}$. (c) and (f) are the linear relationship between the integral desorption charge of oxygen adspecies $Q$ and $v^{-1 / 2}$ at the corresponding Pt nanoelectrodes. 


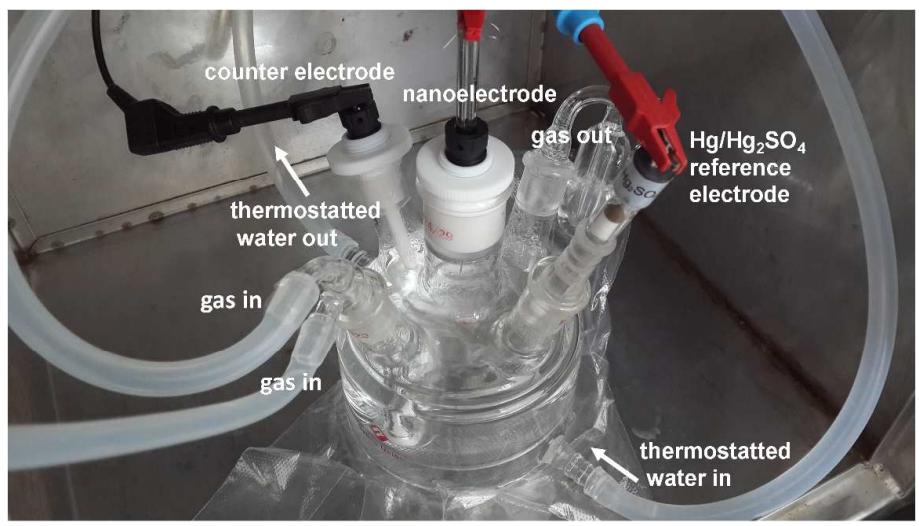

Figure S5 Photograph of a special electrochemical cell with a heat preservation charmber used for the measurement of the surface diffusion coefficient of oxygen adspecies on $\mathrm{Pt}$ surface at different temperatures.
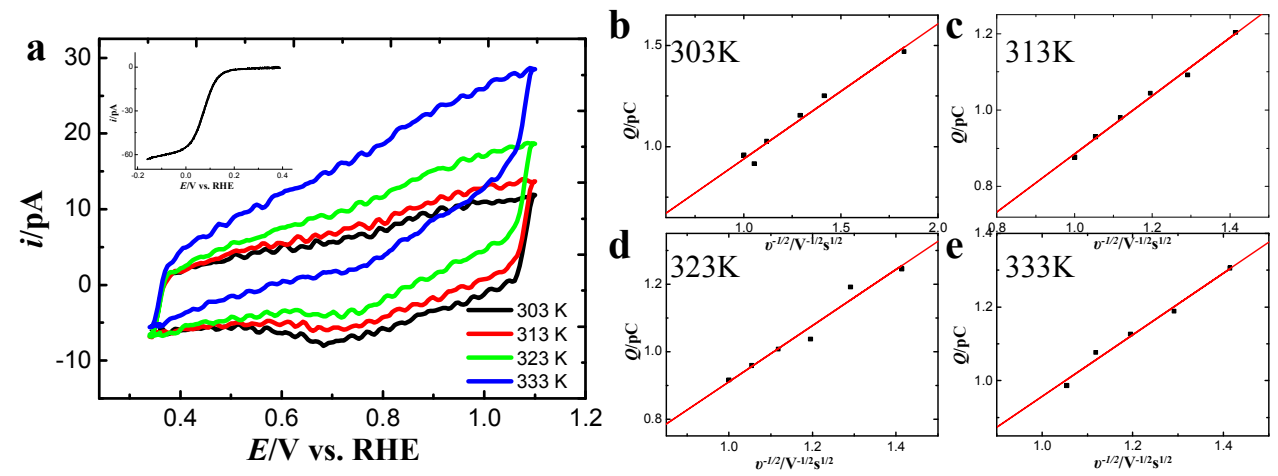

Figure S6 Temperature dependent surface diffusion of oxygen adspecies on Pt nanoelectrodes. (a) Cyclic voltammograms obtained at a 292-nm-radius Pt nanoelectrode in $0.5 \mathrm{M} \mathrm{H}_{2} \mathrm{SO}_{4}$ solution deoxygenated with $\mathrm{Ar}$ at four different temperatures, scan rates are $0.05 \mathrm{~V} \cdot \mathrm{s}^{-1}$. The insert shows $\mathrm{CV}$ with the same electrode in a solution with $2 \mathrm{mM} \mathrm{Ru}\left(\mathrm{NH}_{3}\right)_{6} \mathrm{Cl}_{3}$ and $0.1 \mathrm{M}$ $\mathrm{KCl}$, scan rate is $0.05 \mathrm{~V} \cdot \mathrm{s}^{-1}$; (b)-(e), The linear relationship between the integral desorption charges and the reciprocal of the square root of scanning rate at different temperatures: (b) $303 \mathrm{~K}$, (c) $313 \mathrm{~K}$, (d) $323 \mathrm{~K}$, (e) $333 \mathrm{~K}$. 


\section{S5. Further discussion on surface diffusion}

According to the integral desorption charge at a certain scan rate, the total electroactive area involving surface diffusion region can be obtained. Thereby, the extent of surface diffusion can be further calculated from the following equation:

$$
R F=\left(n_{1} \pi r^{2}+2 \pi r l\right) / \pi r^{2}=n_{1}+2 l_{1} / r
$$

Here, RF is the total roughness factor of the electrode including the contribution of surface diffusion, $n_{1}$ is the "traditional" RF obtained at the electroactive metal area excluding the surface diffusion which is usually no more than 3 for specular disc electrodes, $r$ the radius of the nanoelectrode, $l_{1}$ the distance of surface diffusion. On the macroscopic electrode, $l_{l}$ is much less than $r(l / r<1 \%)$. Thus, the second part caused by surface diffusion is usually neglected. However, when the electrode size is decreased to $100 \mathrm{~nm}$, if the surface diffusion distance is about $2 \mu \mathrm{m}$, the RF caused by surface diffusion is about 20 . This is why NE or even UME can be used to investigate the surface diffusion on electroactive adspecies on the surface of metal catalysts. 


\section{References}

(1) Shao, Y.; Mirkin, M. V.; Fish, G.; Kokotov, S.; Palanker, D.; Lewis, A. Anal. Chem. 1997, 69, 1627.

(2) Sun, P.; Mirkin, M. V. Anal. Chem. 2006, 78, 6526.

(3) Zhan, D.; Velmurugan, J.; Mirkin, M. V. J. Am. Chem. Soc. 2009, 131, 14756.

(4) Bard, A. J.; Faulkner, L. R. Electrochemical Methods Fundamentals and Applications; 2nd Edition ed.; John Wiley \& Sons, Inc: New York, 2001.

(5) Nicholson, R. S.; Shain, I. Anal. Chem. 1964, 36, 706. 